\title{
Ärztliche Führung als (m)eine Profession
}

\author{
Adrian Huber ${ }^{a}$, Yvonne Bogenstätter $^{a}$, Urs Pfefferkorn ${ }^{b}$, Heinz Zimmermann ${ }^{c}$, Emanuela G. Medici \\ ${ }^{a}$ iafob - Institut für Arbeitsforschung und Organsiationsberatung; ${ }^{b}$ Kantonsspital Olten; ${ }^{c}$ emeritierter Chefarzt Inselspital Bern
}

Die (Zusammen-)Arbeit in Spitälern verändert sich. Vermehrte Spezialisierung, Kostenfokussierung und Interdisziplinarität fordern Mitarbeitende wie Kader gleichermassen. Im Umgang mit den aktuellen Herausforderungen im Spital kommt den Leitungspersonen eine besondere Rolle und Verantwortung zu - sie haben zu führen. Doch was bedeutet dies und wie gelingt es, als Arzt oder Ärztin, neben all den anderen Funktionen und Aufgaben, «gut» zu führen?

Eigentlich weiss man (bzw. Mann und Frau), was »Führung» heisst und worauf es grundsätzlich ankommt: Sinn erzeugen, Klarheit schaffen und Entwicklung ermöglichen [1, 2]. Doch wie in so vielem ist auch in Sachen Führung die Theorie klarer als die Praxis und das Papier geduldiger als der Alltag. Worauf kommt es also an? - aufs Tun. Wie verhalte ich mich als Chefarzt

Wie wirksame Mitarbeitendenführung gelingt

Eigentlich gilt es nur das zu tun, was man bereits über wirksame Führung weiss. Mitarbeitende, die sich in ihrem Arbeitsumfeld unwohl fühlen, sind weniger engagiert, während Mitarbeitende mit hoher emotionaler Bindung ans Arbeitsumfeld eher bereit sind, zusätzliche Aufgaben zu übernehmen [3]. Eine entscheidende Ursache für mehr oder minder hohen Grad emotionaler Bindung, für Motivation und Engagement ist die Qualität des Führungsverhaltens der Vorgesetzten [4].

Als professionelle Führungskräfte sollten Kaderärztinnen und Kaderärzte u.a.

- den Umgang mit Mitarbeitenden mögen (und nicht nur ihre Arbeit im Fachgebiet),

- führen wollen (und nicht nur aufgrund der Position führen müssen),

- als Führungskraft (be-)greifbar sein (wissen und sagen was einem wichtig ist, Position beziehen, ansprechbar sein usw.).

Sowie soweit wie situativ möglich

- Sicherheit vermitteln (u.a. durch Transparenz über Ansprüche, Entscheide, Fehler usw.);

- Wertschätzung zeigen (u.a. durch Interesse, Feedback, Anerkennung usw.);

- Fordernd fördern (u.a. durch klare und realistische Ziele, konkrete Vereinbarungen sowie adäquate Unterstützung);

- Sog statt Druck erzeugen (u.a. durch motivierende Perspektiven, Lust auf Veränderungen usw.);

- Handlungsspielräume ermöglichen (u.a. durch Zutrauen, Schaffung von Lernfeldern usw.);

- Ein soziales Betriebsklima schaffen (u.a. durch Ernstnehmen der Wünsche, Bedürfnisse und Sorgen der Mitarbeitenden, vorbildliches Verhalten, Raum für Spass und Humor usw.). gegenüber einem eigensinnigen Oberarzt? Wie stärke ich als Oberärztin die introvertierte Assistenzärztin? Wie vertrete ich als Leitender Arzt eine andere Meinung als meine Chefärztin, ohne dass unsere Arbeitsbeziehung darunter leidet? Und wie gelingt es mir, bei meiner Arbeit gesund zu bleiben? Fragen, auf die sich in der Literatur vielerlei Antworten finden, aber passen diese auch auf mich und meine Arbeitssituation?

\section{Führungskompetenz und -leidenschaft - ein Muss für Kaderärzte im Spital}

Es gibt keine richtige oder falsche Führung, auch im Spital nicht - sondern nur bessere oder weniger gute. Wie man als Führungskraft in ganz bestimmten Situationen agiert, hängt von verschiedenen Faktoren ab, die die Theorie und Führungshandbücher nur begrenzt adaptieren können. Was es braucht, ist "Profession»: 1. Ein adäquates Verständnis von Führung als relevante Funktion und Rolle in der (Zusammen-)Arbeit, 2. durch bewusste Reflexion entwickelte Führungskompetenz sowie 3. eine gehörige Portion Leidenschaft («Lust auf Führung»). Doch wie (ver-)schafft man sich diese Profession?

Ärztinnen und Ärzte - auch in Kaderpositionen - verstehen sich in erster Linie als (hoch) spezialisierte Fachkräfte: Chirurgen, Anästhesistinnen, Mediziner, Orthopädinnen oder Kardiologen. Daneben haben sie eine Position inne, z.B. als Assistenzarzt, Oberärztin, Leitende Ärztin oder Chefarzt. Und mit der Position verbunden sind, nebst den fachbezogenen Aktivitäten und Zuständigkeiten, auch Führungsaufgaben und -verantwortlichkeiten sowie im besseren Falle auch entsprechende Führungskompetenzen. Oft wird Füh- 
rung aber als Sekundärfunktion bzw. Zusatzaufgabe gesehen und wahrgenommen, wofür die meisten Ärztinnen und Ärzte nicht gezielt ausgebildet wurden. Zwar haben Sie im Laufe ihres Werdegangs (mehr oder weniger gute) Führung erfahren und somit eine gewisse Ahnung davon, aber sie kennen weder die grundlegenden Prinzipien und Zusammenhänge, noch effektive Ansätze der Anwendung bzw. deren Wirkung. Meist am eigenen Leibe erfahren wurden jedoch die Auswirkungen von schlechter Führung. Dies ist jedoch ein wenig geeigneter Ansatzpunkt, um Führen zu lernen.

\section{Führungsentwicklung - ein Muss für moderne Spitäler}

Wie lässt sich dies ändern? Wir denken, dass Führung vermehrt als Profession zu verstehen ist. Spitäler brauchen Führung, denn durch sie wird eine effektive und effiziente (Zusammen-)Arbeit ermöglicht. Welche Art der Führung, durch wen und in welcher Form, gilt es situativ zu klären. Damit diese Klärung effektiv erfolgen kann, sind die ärztlichen Führungskräfte und Leitungspersonen der Spitäler entsprechend zu qualifizieren. Ab einer gewissen Position gehört Führung dazu und eine entsprechende Führungsprofession sollte vorhanden sein. Dies bedeutet, Kaderärztinnen und Kaderärzte sollten ein Bewusstsein für ihre besondere Rolle und Verantwortung als Führungskraft entwickeln und leben - wollen, dürfen und können.

Führung sollte Profession sein - aber wie kann ich sie zu meiner machen? Durch die aktive und bewusste Auseinandersetzung mit meinen inneren Bildern, meinen Werten und Zielen sowie mit meiner Situation, meinem Umfeld, seinem und meinem Anspruch an Führung und den Möglichkeiten, die ich habe, (gut) zu führen. Zudem gilt es, sich gute und geeignete Führungsmethoden und -instrumente anzueignen, um in der jeweiligen Situation adäquat und wirkungsvoll agieren zu können.

Spitäler erkennen zunehmend, dass die Förderung dieser bewussten Auseinandersetzung und der damit verbundenen Qualifizierung der ärztlichen Führungskräfte mit einem Mehrwert verbunden sind. Geschulte Kaderärztinnen und Kaderärzte fühlen sich sicherer in ihrer Führungsrolle und gehen dadurch auch anspruchsvolle Führungssituationen aktiv an. Probleme und Konflikte werden eher angesprochen, unbefriedigende Arbeitssituationen vermehrt geklärt und motivierende Arbeitsbedingungen geschaffen - zugunsten einer höheren Qualität und Effizienz in der Leistungserbringung. Und wer will dies nicht?

Literatur

1 Neuberger O. Führen und führen lassen: Ansätze, Ergebnisse und Kritik der Führungsforschung. 2002. (Vol. 2234). utb.

2 Von Rosenstiel L. Mitarbeiterführung in Wirtschaft und Verwaltung. 2002. München: Bayrisches Staatsministerium für Arbeit, Familie und Sozialordnung.

3 Nink, M. Engagement Index Deutschland 2010. 2011; Berlin: Gallup.

4 Information Factory. Schweiz führt?! Eine Studie über Werte, Aufgaben und Wirksamkeit von Führung. 2014; Online unter www.information-factory.com/Schweiz-fuehrt

Führungsseminar für Kaderärztinnen und Kaderärzte In Fortführung der etablierten «biaggi-Führungsseminare», gestaltet das Institut für Arbeitsforschung und Organisationsberatung (iafob) 2015/16 das 10. Führungsseminar für Kaderärztinnen und Kaderärzte.

Dieses Führungsseminar richtet sich an erfahrenere Oberärztinnen und Oberärzte sowie Leitende Ärztinnen und Ärzte, die ihre Führung im Spital reflektieren und ihre Führungskompetenzen gezielt verbessern wollen. Basierend auf konkreten Beispielen vermitteln fachkompetente und spitalumfelderfahrene Expert(inn)en die relevanten Aspekte ärztlicher Führung. Das Seminar ist in vier themenbezogene Module von je 2 Tagen aufgeteilt und bildet in sich ein Ganzes.

2./3.11.2015: Führungsgespräche und Gesprächsführung

7./8.12.2015: Führungsprinzipien und Führungsstil

18./19.1.2016: Teamführung und Konfliktmanagement

3./4.3.2016: Selbstführung und Laufbahngestaltung

Das Führungsseminar ist vom Schweizerischen Institut für Weiter- und Fortbildung (SIWF) anerkannt und wird mit 32 Credits als persönliche Fortbildung angerechnet.

Weitere Informationen und Anmeldung: www.iafob.ch/index. php/veranstaltungen oder adrian.huber[at]iafob.ch 